\title{
Selection for antimicrobial resistance is reduced when embedded in a natural microbial community
}

\author{
Uli Klümper $\mathbb{D}^{1,2} \cdot$ Mario Recker $\mathbb{D}^{3} \cdot$ Lihong Zhang ${ }^{2} \cdot$ Xiaole Yin ${ }^{4} \cdot$ Tong Zhang $^{4} \cdot$ Angus Buckling $^{1} \cdot$ William H. Gaze $^{2}$
}

Received: 28 February 2019 / Revised: 22 July 2019 / Accepted: 23 July 2019 / Published online: 5 August 2019

(c) The Author(s) 2019. This article is published with open access

\begin{abstract}
Antibiotic resistance has emerged as one of the most pressing, global threats to public health. In single-species experiments selection for antibiotic resistance occurs at very low antibiotic concentrations. However, it is unclear how far these findings can be extrapolated to natural environments, where species are embedded within complex communities. We competed isogenic strains of Escherichia coli, differing exclusively in a single chromosomal resistance determinant, in the presence and absence of a pig faecal microbial community across a gradient of antibiotic concentration for two relevant antibiotics: gentamicin and kanamycin. We show that the minimal selective concentration was increased by more than one order of magnitude for both antibiotics when embedded in the community. We identified two general mechanisms were responsible for the increase in minimal selective concentration: an increase in the cost of resistance and a protective effect of the community for the susceptible phenotype. These findings have implications for our understanding of the evolution and selection of antibiotic resistance, and can inform future risk assessment efforts on antibiotic concentrations.
\end{abstract}

\section{Introduction}

The emergence and spread of antimicrobial resistance (AMR) genes in bacterial pathogens has been identified as one of the major threats to human health by the World Health Organisation [1]. Whilst AMR genes have been detected in ancient permafrost samples [2], anthropogenic use of antibiotics has caused a rapid increase in their prevalence [3]. A large body of theory and in vitro work has identified the role of ecological context, such as treatment regime and environmental heterogeneity, in AMR gene dynamics [4-7]. However, the

Supplementary information The online version of this article (https:// doi.org/10.1038/s41396-019-0483-z) contains supplementary material, which is available to authorized users.

Uli Klümper

u.klumper@exeter.ac.uk

1 CLES \& ESI, University of Exeter, Penryn, Cornwall, UK

2 European Centre for Environment and Human Health, University of Exeter Medical School, ESI, Penryn, Cornwall, UK

3 College of Engineering, Mathematics and Physical Sciences, University of Exeter, Penryn, Cornwall, UK

4 Department of Civil Engineering, University of Hong Kong, Hong Kong, China majority of this work has not explicitly considered a crucial feature of microbial ecology: microbes are typically embedded within complex communities of interacting species. This is always the case within human and livestock microbiomes, in which antibiotic-imposed selection is likely to be particularly strong [8]. Here, we combine experiments and theory to determine how selection for AMR is influenced by the presence of other species derived from a natural gut microbial community. The focus of this study is selection for preexisting resistance genes within a focal species, rather than selection on de novo variation arising through spontaneous mutations or acquired through horizontal gene transfer from another species.

Recent experimental studies suggest that selection for AMR genes in complex communities is occurring at antibiotic concentrations (the minimum selective concentration; MSC) that are much lower than those that prevent the growth of susceptible bacteria (minimum inhibitory concentration; MIC) $[9,10]$; as has been previously shown within single species in vitro $[6,7,11]$. However, it is unclear how the presence of other microbial species affects the MSC. While the precise effect of other species is likely context dependent, we hypothesise that the presence of the community will typically increase the MSC. Studies of single species suggest that resistant cells can afford protection to susceptible ones, through both, intracellular and extracellular degradation of 
antibiotics [12-14], thus increasing the relative fitness of susceptible strains and hence the MSC. However, excreted metabolites can both potentiate or decrease antibiotic efficacy, thus decreasing or increasing MSCs [15, 16]. Further, any costs associated with AMR may be enhanced by increased competition for resources, as, for example, has been observed with respect to resistance in flies to parasitoids [17] and bacteria to viruses [18].

To explore the potential effects of community context on AMR selection, we competed isogenic Escherichia coli MG1655 strains, differing exclusively in a single chromosomal resistance determinant, in the presence and absence of a microbial community across a gradient of two different aminoglycoside antibiotics, kanamycin (Kn) and gentamicin $(\mathrm{Gm})$. We embedded the Escherichia coli (E. coli), commonly found in the anaerobic digestive tract of warmblooded mammals [19], within a pig faecal community in experimental anaerobic digesters in an attempt to partially mimic a gut environment. We additionally employed metagenomic analysis, community typing (16S rRNA gene) and mathematical modelling to provide insights into mechanisms underpinning community effects on AMR selection.

\section{Material and methods}

\section{Pig faecal community}

Pig faeces were collected from four Cornish Black pigs without previous exposure to antibiotics in April 2016 on Healey's Cornish Cyder farm (Penhallow, Cornwall, United Kingdom). Two hundred grams of faeces from each pig were pooled, mixed with $400 \mathrm{~mL}$ each of sterile glycerol and $1.8 \mathrm{~g} / \mathrm{L} \mathrm{NaCl}$ solution. The mixture was homogenized for $3 \mathrm{~min}$ in a Retsch Knife mill Gm300 (Retsch $\mathrm{GmbH}$, Haan, Germany) at 2000 rotations per minute (rpm), filtered through a sieve (mesh size $\sim 1 \mathrm{~mm}^{2}$ ), centrifuged at $500 \mathrm{rpm}$ for $60 \mathrm{~s}$ at $4{ }^{\circ} \mathrm{C}$ and the liquid supernatant fraction was collected and frozen at $-80^{\circ} \mathrm{C}$ as the inoculum.

\section{Pig faecal extract}

Two hundred grams of faeces from each pig were pooled, mixed with $800 \mathrm{~mL}$ of sterile $0.9 \mathrm{~g} / \mathrm{L} \mathrm{NaCl}$ solution. The mixture was homogenized for 3 minutes in a Retsch Knife mill Gm300 (Retsch GmbH, Haan, Germany), at 2000 rpm, filtered through a sieve (mesh size $\sim 1 \mathrm{~mm}^{2}$ ) and the liquid fraction was collected. The extract was then centrifuged ( $3500 \mathrm{rpm}, 20 \mathrm{~min}, 4^{\circ} \mathrm{C}$ ), the supernatant collected and autoclaved $\left(121^{\circ} \mathrm{C}, 20 \mathrm{~min}\right)$. The autoclaved extract was centrifuged again (3500 rpm, $20 \mathrm{~min}, 4^{\circ} \mathrm{C}$ ) and the supernatant collected and used as a nutrient supplement.

\section{Strains}

The focal species, E. coli MG1655, was chromosomally tagged with a Tn7 gene cassette encoding constitutive red fluorescence, expressed by the $m$ Cherry gene [20] to ensure that $E$. coli can be detected and distinguished from other community members after competition based on red fluorescence. The $\mathrm{Kn}$ resistant, red fluorescent variant containing resistance gene aph(3')-IIb encoding an aminoglycoside $3^{\prime}$ phosphotransferase was created previously [21, 22].

To create the $\mathrm{Gm}$ resistant mutant the strain was further tagged through electroporation with the pBAM delivery plasmid containing the mini-Tn5 delivery system [23, 24] for $\mathrm{Gm}$ resistance gene aac $\mathrm{Cl}$ encoding a $\mathrm{Gm} 3^{\prime}-\mathrm{N}$-acetyltransferase [25]. Successful clones were screened for $\mathrm{Gm}$ resistance $(30 \mu \mathrm{g} / \mathrm{mL})$ and for the chosen clone a single strain growth curve in lysogeny broth (LB) was measured to ensure that the cost of the resistance gene was lower than $10 \%$ compared with the susceptible strain to ensure competitive ability.

\section{Competition experiments}

Competition experiments as well as initial growth of focal species strains were performed in $25 \mathrm{~mL}$ serum flasks with butyl rubber stoppers. As growth medium $10 \mathrm{~mL}$ of sterile LB medium supplemented with $0.1 \%$ pig faecal extract, $50 \mathrm{mg} / \mathrm{L}$ Cysteine- $\mathrm{HCl}$ as an oxygen scrubber and $1 \mathrm{mg} / \mathrm{L}$ resazurin as a redox indicator to ensure anaerobic conditions [26], was added to each reactor, heated in a water bath to $80^{\circ} \mathrm{C}$ and bubbled with oxygen-free $\mathrm{N}_{2}$ gas until the oxygen indicator resazurin turned colourless. After cooling down to $37^{\circ} \mathrm{C}$ the appropriate concentration of antibiotic was added from a $1000 \times$ anaerobic stock solution.

Two isogenic pairs of the focal species, the susceptible, red fluorescent $E$. coli strain with either its $\mathrm{Gm}$ or $\mathrm{Kn}$ resistant counterpart, were competed across a gradient of six antibiotics concentrations $(\mathrm{Gm}[\mu \mathrm{g} / \mathrm{mL}]: 0,0.01,0.1,1,10$, 100; Kn [ $\mu \mathrm{g} / \mathrm{mL}]: 0,0.02,0.2,2,20,200)$. Strains as well as the community ( $100 \mu \mathrm{L}$ of frozen stock) were grown separately under anaerobic conditions in triplicate reactors, replicates were combined, harvested through centrifugation, washed twice in $0.9 \%$ anaerobic $\mathrm{NaCl}$ solution and finally resuspended in $0.9 \% \mathrm{NaCl}$ solution, adjusted to $\mathrm{OD}_{600} 0.1$ $\left(\sim 10^{7}\right.$ bacteria/mL) and subsequently used in competition experiments. While the community was grown as an inoculum from the same frozen, homogenized stock, both subsampling and cultivation bias, inherent when growing an environmental community under laboratory conditions led to differences in original composition of the model community (Figs. S1, S2). When growing the community in isolation in the absence of antibiotics carrying capacity was reached after $18 \mathrm{~h}$ based on $\mathrm{OD}_{600}$ readings in a spectrophotometer. 
Isogenic strains were mixed at 1:1 ratio (community absent treatment), and that mix further added at $10 \%$ ratio to $90 \%$ of the faecal community (community present treatment). Approximately $10^{6}$ bacteria of either mix were transferred to six replicate reactors of each of the antibiotic concentrations and grown at $37{ }^{\circ} \mathrm{C}$ with $120 \mathrm{rpm}$ shaking for $24 \mathrm{~h}$, which allowed growth up to carrying capacity. As a consequence of normalizing the total inoculum size the resulting inoculum size of the focal species in absence $\left(\sim 10^{6}\right.$ bacteria) and presence $\left(\sim 10^{5}\right.$ bacteria $=10 \%$ of total inoculum) of the community differed. A volume of $100 \mu \mathrm{L}$ of each reactor was then transferred to a fresh bioreactor, grown for $24 \mathrm{~h}$, transferred again for a final $24 \mathrm{~h}$ growth cycle and finally harvested for subsequent analysis.

\section{Fitness assay}

From each reactor after 3 days $\left(\mathrm{T}_{3}\right)$, as well as the inocula $\left(\mathrm{T}_{0}\right)$, a dilution series in sterile $0.9 \% \mathrm{NaCl}$ solution was prepared and plated on $\mathrm{LB}$ and $\mathrm{LB}+\mathrm{AB}(30 \mu \mathrm{g} / \mathrm{mL} \mathrm{Gm}$ or $75 \mu \mathrm{g} / \mathrm{mL} \mathrm{Kn}$ ). For appropriate dilutions total and resistant red fluorescent $E$. coli colonies were counted under the fluorescence microscope. Plating of the susceptible strain on $\mathrm{LB}+\mathrm{AB}$ plates further did not lead to any growth of spontaneous mutants. The relative fitness $(\rho)$ of the resistant ( $r$ ) compared with the susceptible strain $(s)$ strain was subsequently calculated based on their individual growth rate $(\gamma)$ throughout the competition experiment:

$$
\begin{aligned}
\rho & =\frac{\gamma_{r}}{\gamma_{s}}=\frac{\log \left(10^{6} \times n_{r}^{T_{3}} / n_{r}^{T_{0}}\right)}{\log \left(10^{6} \times n_{s}^{T_{3}} / n_{s}^{T_{0}}\right)} \\
& =\frac{\log \left(10^{6} \times n_{r}^{T_{3}} / n_{r}^{T_{0}}\right)}{\log \left(10^{6} \times\left(n_{\text {total }}^{T_{3}}-n_{r}^{T_{3}}\right) /\left(n_{\text {total }}^{T_{0}}-n_{r}^{T_{0}}\right)\right)}
\end{aligned}
$$

Statistical significance testing $(n=6)$ was performed using a one-tailed $t$-test against neutral selection $(\rho=1)$ and ANOVA corrected for multiple testing to compare the relative fitness of different samples.

\section{MIC assay}

To assess the MIC of the susceptible and the resistant focal strain individually in the presence and absence of the microbial community reactors were inoculated with $10^{5}$ of the focal bacteria and $10^{6}$ bacteria from the community for the community present treatment. Triplicate reactors were grown overnight across a gradient of antibiotics. Concentrations were increased by $1 \mu \mathrm{g} / \mathrm{mL}$ (Gm susceptible), $2 \mu \mathrm{g} / \mathrm{mL}$ (Km susceptible), $25 \mu \mathrm{g} / \mathrm{mL}$ (Gm resistant) and $50 \mu \mathrm{g} / \mathrm{mL}$ (Km resistant), respectively. Reactors were then harvested and plated out on LB agar. Positive growth was scored as more than fourfold bacterial colonies growing on the plates compared with plating of the inoculum. The MIC was defined as the first concentration at which no positive growth was observed.

\section{DNA extraction and sequencing}

Bacteria from each reactor, as well as inoculum and original pig faecal community were harvested through centrifugation of $2 \mathrm{~mL}$ of liquid, followed by DNA extraction using the Qiagen PowerSoil kit as per the manufacturer's instructions. The quality and quantity of the extractions was confirmed by $1 \%$ agarose gel electrophoresis and dsDNA BR (Qubit), respectively.

16S rRNA gene libraries were constructed using multiplex primers designed to amplify the V4 region [27]. Amplicons were generated using a high-fidelity polymerase (Kapa 2G Robust), purified with the Agencourt AMPure XP PCR purification system and quantified using a fluorometer (Qubit, Life Technologies, Carlsbad, CA, USA). The purified amplicons were pooled in equimolar concentrations based on Qubit quantification. The resulting amplicon library pool was diluted to $2 \mathrm{nM}$ with sodium hydroxide and $5 \mathrm{~mL}$ were transferred into $995 \mathrm{~mL} \mathrm{HT1}$ (Illumina) to give a final concentration of $10 \mathrm{pM}$. Six hundred millilitres of the diluted library pool was spiked with $10 \%$ PhiXControl v3 and placed on ice before loading into Illumina MiSeq cartridge following the manufacturer's instructions. The sequencing chemistry utilized was MiSeq Reagent Kit v2 (500 cycles) with run metrics of 250 cycles for each paired end read using MiSeq Control Software 2.2.0 and RTA 1.17.28.

Metagenomic libraries were created using the KAPA high throughout Library Prep Kit (Part no: KK8234) optimized for $1 \mu \mathrm{g}$ of input DNA with a size selection and performed with Beckman Coulter XP beads (Part no: A63880). Samples were sheared with a Covaris S2 sonicator (available from Covaris and Life Technologies) to a size of $350 \mathrm{bp}$. The ends of the samples were repaired, the $3^{\prime}-5^{\prime}$ exonuclease activity removed the $3^{\prime}$ overhangs and the polymerase activity filled in the $5^{\prime}$ overhangs creating blunt ends. A single 'A' nucleotide was added to the $3^{\prime}$ ends of the blunt fragments to prevent them from ligating to one another during the adapter ligation reaction. A corresponding single ' $\mathrm{T}$ ' nucleotide on the 3 ' end of the adapter provided a complementary overhang for ligating the adapter to the fragment ensuring a low rate of chimera formation. Indexing adapters were ligated to the ends of the DNA fragments for hybridisation on a flow cell. The ligated product underwent size selection using the XP beads detailed above, thus removing the majority of unligated or hybridized adapters. Prior to hybridisation the samples underwent six cycles of PCR to selectively enrich those DNA fragments with adapter molecules on both ends and to amplify the amount of DNA in the library. The PCR 
was performed with a PCR primer cocktail that anneals to the ends of the adapter. The insert size of the libraries was verified by running an aliquot of the DNA library on a PerkinElmer GX using the High Sensitivity DNA chip (Part no: 5067-4626) and the concentration was determined by using a High Sensitivity Qubit assay. All raw sequencing data have been submitted to ENA under study accession number PRJEB29924.

\section{S rRNA gene analysis}

Sequence analysis was carried out using mothur v.1.32.1 [28] and the MiSeq SOP [27] as accessed on 07.08.2017 on http://www.mothur.org/wiki/MiSeq_SOP. Sequences were classified based on the RDP classifier [29]. Diversity was assessed based on observed OTUs at $97 \%$ sequence similarity. All sequences of the focal species E. coli were removed based on $\geq 99 \%$ sequence similarity. No sequences with this degree of similarity to the focal species were detected in the original faecal community. NMDS plots for the community were created based on the Bray-Curtis dissimilarity metric [30].

Further sample similarity was tested using analysis of molecular variance (AMOVA) a nonparametric analogue of traditional ANOVA testing. AMOVA is commonly used in population genetics to test the hypothesis that genetic diversity between two or more populations is not significantly different from a community created from stochastically pooling these populations [31, 32].

\section{Metagenomic analysis}

Metagenomic samples, as well as a reference genome for the focal species E. coli MG1655, were analysed using the ARG-OAP pipeline for antibiotic resistance genes detection from metagenomic data using an integrated structured antibiotic resistance gene database [33]. This resulted in the abundance of different resistance gene classes and subtypes within these groups normalized by $16 \mathrm{~S}$ rRNA gene copy number. Antibiotics resistance genes detected in the E. coli reference genome were subtracted from the total number of hits per 16S rRNA gene copy based on the abundance of $E$. coli 16S rRNA gene/total 16S rRNA gene. Further, all antibiotics resistance gene numbers were normalized to the amount of pig faecal community 16S rRNA gene per total 16S rRNA gene copy.

\section{Mathematical model}

In order to illustrate possible mechanisms underlying the data for bacterial fitness in the presence/absence of the community for varying concentrations of $\mathrm{Gm}$ and $\mathrm{Kn}$, we described our experimental setup mathematically. For this we first developed a discrete-time mathematical model for the growth of the susceptible and drug-resistant bacteria, $s$ and $r$, respectively, in the presence or absence of the community, $c$.

\section{Bacterial growth}

The discrete-time model describing the growth of the bacteria $i, i=s, r, c$, is governed by the following iterative model

$$
n_{i}^{t+1}=n_{i}^{t}\left(1+\phi_{i}\left(1-g_{i}\right)\left(1-f_{i}\right)\right)
$$

where $n_{i}^{t+1}$ is the size of the population of strain $i$ at time $t+1$, and $\phi_{i}$ is the maximum growth rate in the absence of competition and drug pressure. The reduction in growth due to density-dependent regulation/resource limitation, given as

$g_{i}=\frac{\sum_{j} e_{i j} n_{j}}{k_{d}}$,

with $k_{d}$ as the carrying capacity and $e_{i j}$ being the competition coefficient, describing how much the presence of an allospecific strain $j$ impacts the competitive fitness of strain $i$. The reduction in bacterial growth due to drug pressure, $f_{i}$, is governed by a generalised logistic function

$f_{i}=\min \left(f_{\max }, \frac{1}{1+e^{\alpha_{i}-\beta_{i} \ln c}}\right)$,

where $c$ is the drug concentration (in $\mu \mathrm{g} / \mathrm{mL}$ ), $\alpha_{i}$ and $\beta_{i}$ are the parameters describing the dose-response relationship for strain $i$, and $f_{\max }=0.9$ is the maximum growth inhibition.

\section{Model simulation and relative fitness calculation}

Starting from an initially small number of bacteria in fresh medium, we ran the model for 30 generations, at which point the bacterial population had reached carrying capacity, and diluted the population accordingly. The bacteria were again allowed to grow for 30 generations before being diluted and grown for a final 30 generations. At this point we calculated the relative fitness of the resistant strain as

$\rho=\frac{\gamma_{r}}{\gamma_{s}}=\frac{\log \left(10^{6} \times n_{r}^{90} / n_{r}^{0}\right)}{\log \left(10^{6} \times n_{s}^{90} / n_{s}^{0}\right)}$.

\section{Community-dependent change in drug resistance/ susceptibility}

The Kn data seem to suggest that the benefit of the drug resistant bacteria is reduced in the presence of the 
community at medium to high drug concentrations pointing towards a decrease in the susceptibility of the susceptible strain in a community context. We captured this scenario by making the dose-response parameters $\alpha_{s, r}$ and $\beta_{s, r}$ explicitly dependent on the density of the community by increasing the resistance of susceptible strain, $s$, i.e.

$$
\begin{aligned}
& \alpha_{s}(t)=\alpha_{s, 0}\left(1+\frac{1.3 n_{c}^{t}}{n_{c}^{t}+10^{3}}\right), \\
& \beta_{s}(t)=\beta_{s, 0}\left(1+\frac{0.35 n_{c}^{t}}{n_{c}^{t}+10^{3}}\right),
\end{aligned}
$$

where $\alpha_{i, 0}$ and $\beta_{i, 0}$ are the time-independent dose-response parameters. The effect of density dependence is further illustrated (Fig. S3).

\section{Parameter estimations}

For each drug ( $\mathrm{Gm}$ and $\mathrm{Kn}$ ) we obtained a set of parameter values that resulted in a good overall fit between the model simulations and the data, where the data comprised the observed relative fitness for both sets of experiments (i.e. bacteria grown in the presence and absence of the community) for six different drug concentrations. To allow for logarithmic regression the non-antibiotic control was assumed as one order of magnitude lower than the lowest concentration used in the experiment. The parameter values were determined by minimising the rootmean-square error using an optimisation algorithm akin to simulated annealing [34]. The aim here was not to perform rigorous parameter estimation but rather to find a set of parameters that, given specific model constraints and assumptions, resulted in model behaviours that qualitatively agreed with both the observed dynamics over the repeated growth cycles and the empirically determined fitness values. In fact, our method failed to find a unique set of values that consistently gave the best fitting model, which suggests that the available data was insufficient to determine the global maximum. However, the qualitative

Table 1 Model parameter values for gentamicin selection curves

\begin{tabular}{lllll}
\hline Gentamicin & & & & \\
\hline Parameter & All & Susceptible & Resistant & Community \\
\hline$\varphi$ & & 1.4 & 1.3 & 1.3 \\
$e_{i j}$ & & 1 & 2.3 & 3 \\
$\alpha_{\mathrm{i}, 0}$ & & 1.3 & 2.9 & 1.6 \\
$\beta_{\mathrm{i}, 0}$ & & 0.7 & 0.8 & 0.6 \\
$k_{d}$ & $10^{5}$ & - & - & - \\
$f_{\max }$ & 0.9 & - & - & - \\
\hline
\end{tabular}

Table 2 Model parameter values for kanamycin selection curve

\begin{tabular}{lllll}
\hline Kanamycin & & & & \\
\hline Parameter & All & Susceptible & Resistant & Community \\
\hline$\varphi$ & - & 1.9 & 1.8 & 1.3 \\
$e_{i j}$ & - & 1.7 & 1.3 & 1.6 \\
$\alpha_{\mathrm{i}, 0}$ & - & 1.0 & 1.4 & 1.6 \\
$\beta_{\mathrm{i}, 0}$ & - & 0.6 & 0.4 & 0.5 \\
$k_{\mathrm{d}}$ & $10^{5}$ & - & - & - \\
$f_{\max }$ & 0.9 & - & - & - \\
\hline
\end{tabular}

relationships between individual parameters and between the parameters comparing the two antimicrobials were fairly consistent between model runs. Tables 1, 2 list the sets of parameters estimated for the two different antibiotics. These parameters allow changes in community and focal species densities to be estimated throughout the individual competition experiments based on start- and end-point measurements (Fig. S4).

\section{Results}

\section{Community context affects selection for gentamicin resistance}

Isogenic strains of the focal species $E$. coli, with and without $\mathrm{Gm}$ resistance, were competed in the presence and absence of a pig faecal community across a 5 orders of magnitude gradient of $\mathrm{Gm}$ concentrations. Independent of antibiotic concentration the focal species increased in abundance during the 3 day competition experiment from $\sim 10 \%$ at inoculation to above $90 \%$ relative abundance based on 16S rRNA gene sequencing (Fig. S5A and B). Both resistant and sensitive strains, as well as the community, showed positive growth across the whole range of concentrations and both treatments with focal species' cell counts increasing by $2.25-3.96$ orders of magnitude per day (Fig. 1a).

There was a small competitive fitness cost ( $t$-test against 1 , $p=0.0005)$ of $\mathrm{Gm}$ resistance in the absence of the community $\left(\rho_{\mathrm{r}}=0.955 \pm 0.014\right.$, mean $\left.\pm \mathrm{SD}\right)$, and this cost appeared to be greatly increased when the community was present (Fig. 2) $\left(\rho_{\mathrm{r}}=0.788 \pm 0.016\right)$ (ANOVA corrected for multiple testing, $p<0.01, F=360.36$ ). At all concentrations between 0 and $10 \mu \mathrm{g} / \mathrm{mL}$ the susceptible strains relative growth benefited more from presence of the community when compared with the resistant one (ANOVA corrected for multiple testing, $p<$ 0.05 ), until at $100 \mu \mathrm{g} / \mathrm{mL} \mathrm{Gm}$, the community had no significant effect on relative growth (ANOVA corrected for multiple testing, $p=0.259, F=1.42$ ) (Fig. 1b). 

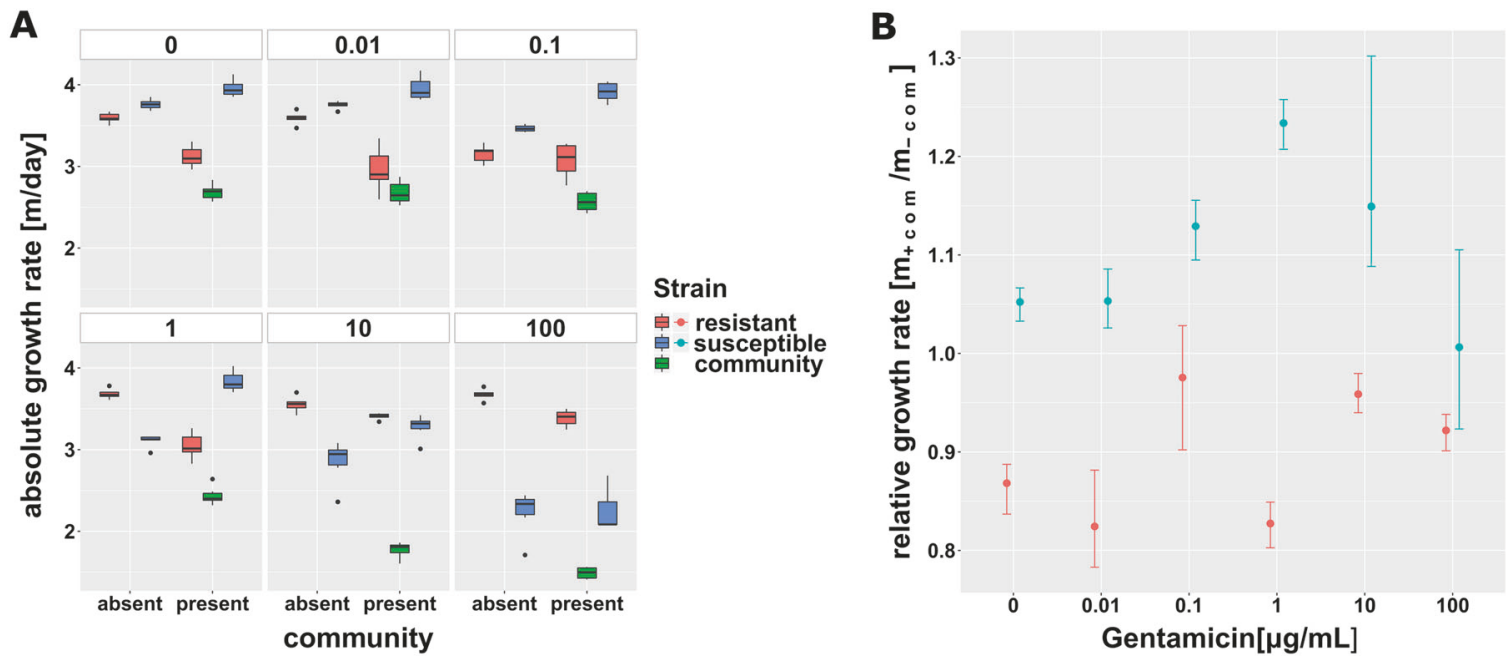

Fig. 1 Malthusian growth parameter per day of the focal species' isogenic strains for gentamicin. Values are displayed across the antibiotic gradient and in absence and presence of the gut microbial community. a Average $( \pm \mathrm{SD}, n=6)$ logarithmic absolute growth per day for the resistant strain, the susceptible strain and the community. A different inoculum size of the focal species in absence $\left(\sim 10^{6}\right.$

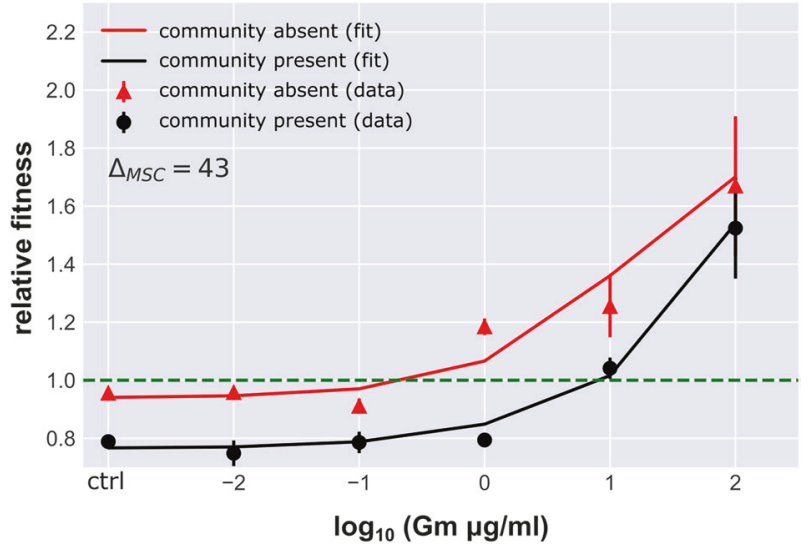

Fig. 2 Relative fitness of the gentamicin resistant strain. Values (mean $\pm \mathrm{SD}, n=6$ ) in presence (black) and absence (red) of the community. Solid lines represent the best fit fitness curve through the mathematical model based on parameter estimates presented in Table 1 . The dashed line indicates neutral selection at a relative fitness of $\rho_{\mathrm{r}}=1$, where the intercept with the fitness curve indicates the minimal selective concentration

\section{Community composition is altered across the gentamicin gradient}

It is possible that changes in community composition across the antibiotic gradient may have contributed to the observed changes in selection for resistance caused by the community, notably between 10 and $100 \mu \mathrm{g} / \mathrm{mL}$. The composition of the microbial community changed significantly from the collected faecal sample, to inoculum and further during the duration of the experiment (AMOVA, $p<0.001$, Fig. S1A). Above $1 \mu \mathrm{g} / \mathrm{mL} \quad \mathrm{Gm}$ the previously dominant bacteria) and presence $\left(\sim 10^{5}\right.$ bacteria $=10 \%$ of total inoculum $)$ of the community was used. b Ratio of absolute Malthusian growth parameters (with 95\% confidence intervals based on 1000-fold bootstrap analysis) in presence and absence of the microbial community across the gradient of antibiotic concentrations

Proteobacteria were outcompeted by Firmicutes (Fig. S1B) leading to a significant (AMOVA, $p<0.01$ ) separation of communities below and above this threshold concentration in the NMDS plot (Fig. S1A). However, there was no significant change in composition between 10 and $100 \mu \mathrm{g} / \mathrm{mL}$, suggesting that compositional changes did not play a major role in community-imposed selection.

\section{Community context imposes a cost of resistance}

To test the hypotheses derived from the numerical data we used numerical simulations of our experimental set up to determine the likely mechanisms underpinning the observed population dynamics in a common logarithmic growth model. We determined models based on the key empirical findings in the absence of the community (specifically, that there is a cost of resistance in the absence of antibiotics, and that antibiotics inhibit the growth of the sensitive strain in a dose dependent manner), and then determined the most parsimonious way, in which the community could have altered the relative fitness of the resistant and susceptible strains (Table 1). We found a good fit to the data simply by assuming that the community imposed a greater competitive effect, constant across the antibiotic gradient, on the resistant rather than the sensitive strain $\left(e_{r j}>>e_{c j}>e_{s j}\right.$; where $e_{i j}$ is the competition coefficient imposed on the focal population (resistant $r$, susceptible $s$ and community $c$ ) by the community). Further, higher concentrations of Gm result in a drastic drop in the community's growth rate, and hence a reduction of the elevated cost of resistance imposed by the community at these higher levels of antibiotic due to 
Table 3 MIC of the focal species, in isolation in presence and absence of the community. The range of highest growth concentration to minimal tested inhibitory concentration is shown

\begin{tabular}{llll}
\hline Antibiotic & Strain & Community & MIC $[\mu \mathrm{g} / \mathrm{mL}]$ \\
\hline Gentamicin & Susceptible & Absent & $8-9$ \\
& & Present & $8-9$ \\
& Resistant & Absent & $100-125$ \\
& & Present & $100-125$ \\
Kanamycin & Susceptible & Absent & $16-18$ \\
& & Present & $18-20$ \\
& Resistant & Absent & $>800$ \\
& & Present & $>800$ \\
\hline
\end{tabular}

reduced competition. This in combination with very little growth of the susceptible strain explains why the relative fitness between the community present and community absent treatments converged at high antibiotic concentrations $(100 \mu \mathrm{g} / \mathrm{mL})$ with relative fitness primarily determined by the growth of the resistant strain.

The numerical simulation allowed us to estimate the change in MSC from absence to presence of the community by deterministically evaluating the concentration at the intercept with neutral selection at a relative fitness of $\rho_{\mathrm{r}}=1$. We estimated a 43-fold increase in MSC in the presence of the community (Fig. 2). The MIC for the susceptible strain remained stable at $8-9 \mu \mathrm{g} / \mathrm{mL}$ in both presence and absence of the community (Table 3). Consequently, this 43-fold increase in MSC shifts selection from concentrations far below the MIC in absence to concentrations around the MIC in presence of the community.

\section{Community context affects selection for kanamycin resistance}

As with $\mathrm{Gm}$, the focal species increased in abundance during the 3 day competition experiment from $\sim 10 \%$ at inoculation to above 90\% relative abundance (Fig. S5C and D). Again, both strains, as well as the community, increased in abundance across both treatments and all concentrations of the 5 orders of magnitude antibiotic gradient with focal species' cell numbers increasing by 1.45-3.09 orders of magnitude per day (Fig. 3a). In the absence of this community, Kn resistance also imposed a slight metabolic fitness cost on the resistant strain $\left(\rho_{\mathrm{r}}=0.915 \pm 0.036\right)$ (Fig. 3b).

However, unlike $\mathrm{Gm}$, the community did not increase the general cost of resistance. Indeed, the community had no significant effect on the relative fitness of the resistant strain except at a concentration of $20 \mu \mathrm{g} / \mathrm{mL}$ (ANOVA corrected for multiple testing, $p=0.002, F=15.58$ ) (Fig. 4). There was a clear fitness advantage for the resistant strain in the absence of the community at this concentration $\left(\rho_{\mathrm{r}}=1.288\right.$ $\pm 0.149 ; t$-test against $1, p=0.0052$ ), while in the presence of the community, this difference in relative fitness, though still significant ( $t$-test against $1, p=0.0088)$, was considerably lower $\left(\rho_{\mathrm{r}}=1.034 \pm 0.020\right)$. At $200 \mu \mathrm{g} / \mathrm{mL} \mathrm{Kn}$, close to the susceptible strains MIC, the resistant strain had an equally high relative fitness regardless of the presence of the community (ANOVA corrected for multiple testing, $p=0.079, F=3.84$ ).

Community and antibiotic resistance composition remain stable across the kanamycin gradient

As with the Gm experiment, a significant shift in community composition from collected faecal sample, to inoculum and further during the duration of the $\mathrm{Kn}$ experiment (AMOVA, $p<0.001$, Fig. S2A) was observed. However, across the whole gradient of antibiotics, Firmicutes (Fig. S2B) remained the dominant phylum with no significant changes in community composition as a result. As such, compositional changes again cannot explain the impact of the community on focal strain fitness under selection at $20 \mu \mathrm{g} / \mathrm{mL}$ only. We additionally carried out metagenomic analysis for the 0,2 and $20 \mu \mathrm{g} / \mathrm{mL} \mathrm{Kn}$ treatments to determine whether relative abundance of resistance genes had changed within the community, despite the fact that there were no changes in community composition. Resistance to aminoglycoside (ANOVA, $p=0.04$ ) and other classes of antibiotics (fosmidomycin, kasugamycin, macrolides, polymyxin and tetracycline (ANOVA, all $p<$ $0.01)$ ) significantly increased in the community of all reactors compared with the original faecal community independent of antibiotic concentrations (Fig. 5a). However, there was no significant difference between Kn concentration and the abundance (ANOVA, $p=0.15$ ) of aminoglycoside resistance in general (Fig. 5a) or any specific aminoglycoside resistance subtypes (Fig. 5b) suggesting that relative fitness of the focal species was not influenced by the community resistome. Resistance genes detected by metagenomic analysis were however expressed, since resistant colonies from community members were detected on selective plates after the competition experiment. Unsurprisingly, since no antibiotic concentrationdependent selection for aminoglycoside resistance was observed within the community, no significant co-selection for resistance to any other classes of antibiotics was observed either.

Presence of the community can enhance growth of susceptible E. coli population at intermediate antibiotic concentrations

Numerical simulations showed that, unlike for Gm resistance, a community-imposed increase in the cost of $\mathrm{Kn}$ resistance was unable to explain why the benefit to the drug resistant focal $E$. coli strain was reduced in the presence of the community at intermediate drug concentrations $\left(e_{r j}=e_{s}\right)$. This suggested different interactions between $E$. coli and the rest of 
A

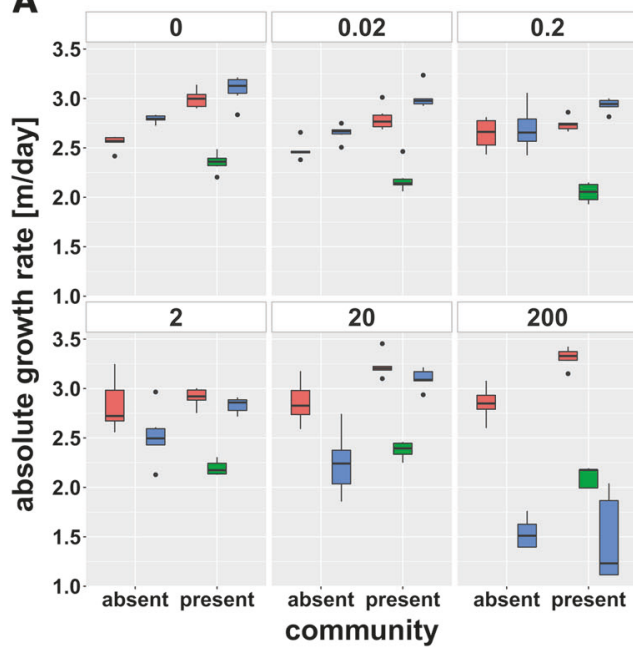

Fig. 3 Malthusian growth parameter per day of the focal species' isogenic strains for kanamycin. Values are displayed across the antibiotic gradient and in absence and presence of the gut microbial community. a Average $( \pm \mathrm{SD}, n=6)$ logarithmic absolute growth per day for the resistant (red), the susceptible strain and the community. A different inoculum size of the focal species in absence $\left(\sim 10^{6}\right.$

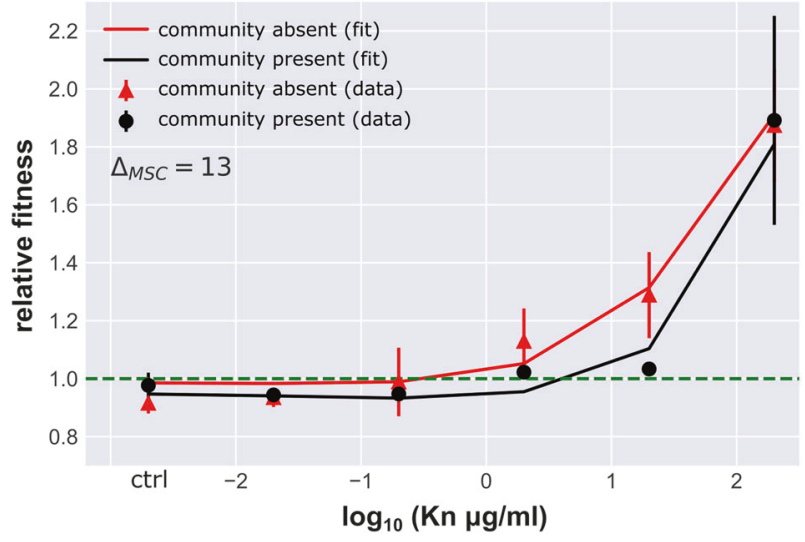

Fig. 4 Relative fitness of the kanamycin resistant strain. Values (mean $\pm \mathrm{SD}, n=6$ ) in the presence (black) and absence (red) of the community. Solid lines represent the best fit fitness curve through the mathematical model based on parameter estimates presented in Table 2. The dashed line indicates neutral selection at a relative fitness of $\rho_{\mathrm{r}}=1$, where the intercept with the fitness curve indicates the minimal selective concentration

the community, and we speculated that the community might have provided a protective effect against $\mathrm{Kn}$ for the susceptible E. coli. Growth data demonstrated this to be the case: in the community treatment the growth rate of both the susceptible and resistant $E$. coli was altered in a consistent fashion across the lower concentrations. The only exceptions was observed at $20 \mu \mathrm{g} / \mathrm{mL}$, where the growth rate of the susceptible, but not the resistant strain, was significantly increased by the presence of the community (ANOVA corrected for multiple testing, $p=0.002$, $F=15.58$ ) (Fig. 3b). Further, a slight increase in the MIC of

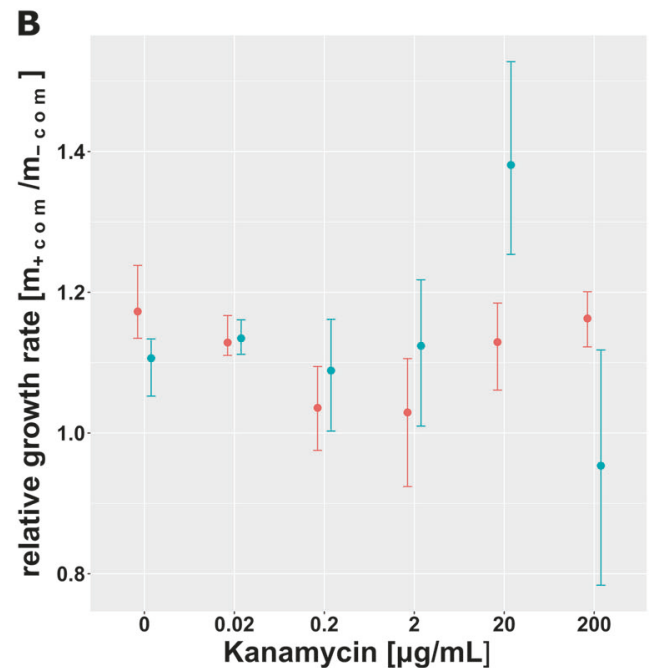

bacteria) and presence $\left(\sim 10^{5}\right.$ bacteria $=10 \%$ of total inoculum $)$ of the community was used. b Ratio of absolute Malthusian growth parameters (with $95 \%$ confidence intervals based on 1000-fold bootstrap analysis) in the presence and absence of the microbial community across the gradient of antibiotic concentrations

the susceptible strain in the presence of the community from $16-18$ to $18-20 \mu \mathrm{g} / \mathrm{mL}$ (Table 3) supports the notion of a protective effect. We investigated if a protective effect of the community was sufficient to explain the observed data by fitting numerical simulations where the dose-response parameters $\alpha_{s, r}$ and $\beta_{s, r}$ were explicitly dependent on the timedependent density of the community (as listed in Table 2). The resulting model provided a good fit to the experimental data, suggesting that community protection was driving the observed population dynamics with a 12-fold increase in MSC. Despite this MSC shift, other than for Gm resistance for Kn selection was still observed at concentrations 5-10fold below the MIC of the susceptible strain.

\section{Discussion}

In this study we investigated how being embedded within a semi-natural community (a pig gut derived community in an anaerobic digester) affects selection for AMR within a focal species (E. coli). For two antibiotics ( $\mathrm{Gm}$ and $\mathrm{Kn})$, we find the presence of the community selects against resistance, resulting in 1-2 orders of magnitude higher minimal selective concentrations for antibiotic resistance and thus achieving selection either closer towards or for $\mathrm{Gm}$ even at around the susceptible strains MIC. This suggests that recent in vitro single strain based estimates of MSCs $[6,7,11]$ are likely much lower than would be observed in vivo and might explain why in certain ecosystems no selection for antibiotic resistance was observed in focal strains [35]. 
A

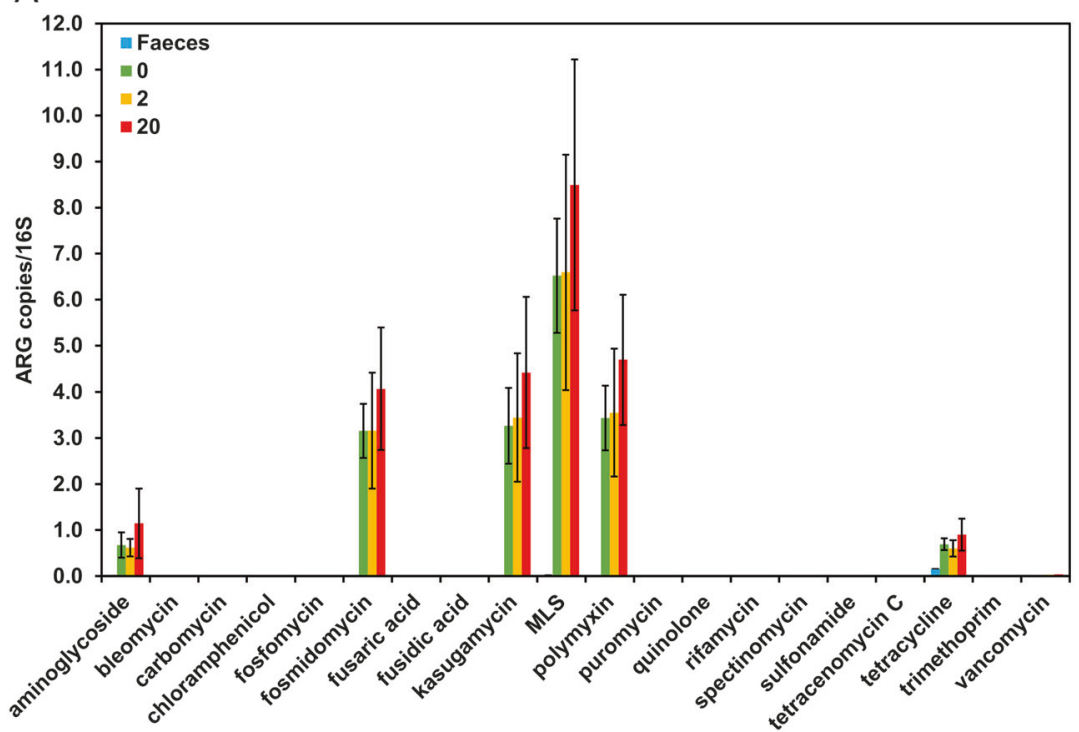

$B$

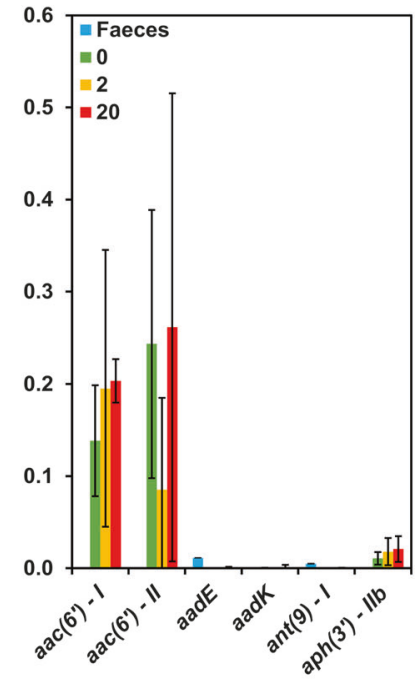

Fig. 5 Detected resistance genes. Type (a) and aminoglycoside subtype (b) relative abundance (resistance gene number normalized with $16 \mathrm{~S}$ rRNA gene copy number), in original faecal community and in final reactor community at three kanamycin concentrations (mean \pm
$\mathrm{SD} ; n_{\mathrm{faeces}}=2, n_{\mathrm{Kn} 0}=6, n_{\mathrm{Kn} 2}=6 n_{\mathrm{Kn} 20}=5$ ). Only genes detected with the ARGs-OAP pipeline are shown. MLS = Macrolides, Lincosamides, Streptogramines

protective effects have been reported extensively within species $[13,14]$, as well as more recently within more complex communities [12], either because of extra- or intracellular modification of antibiotics. Other common mechanisms known to increase a strains resistance to antibiotics in communities involve flocculation [40] or biofilm formation [41, 42], but might here only play a minor role due to the shaking conditions.

A main difference between the $\mathrm{Kn}$ and $\mathrm{Gm}$ competition experiments was observed that could have implications for the likely mechanisms underpinning community-mediated changes in selection for resistance. In the absence of antibiotic selection, a higher community diversity involving a larger proportion of Proteobacteria was detected in the competing community during the $\mathrm{Gm}$ experiment compared with the Kn experiment. Since the focal species E. coli also belongs to the Proteobacteria, the difference in community structure and especially proteobacterial abundance could have increased the level of niche overlap between focal strain and community and thus competition [43], which may explain why $\mathrm{Gm}$ resistance had a significantly increased cost while $\mathrm{Kn}$ resistance did not.

The two general mechanisms discussed above all underlie selection for standing variation in pre-existing resistance genes, rather than selection on de novo variation arising through spontaneous mutations or horizontal gene transfer from other species. For de novo chromosomal mutations, the community is likely to further limit the spread of resistance, because the reduced population sizes of the focal strains in the presence of the community increase the chance that more 
costly mutations will be fixed [44]. In contrast, being embed in a community might enhance the spread of resistance. First, there will be a greater source of resistance genes available to the focal species. Second, selection against resistance acquired through horizontal gene transfer at low antibiotic concentrations might follow different dynamics. While chromosomal resistance might be outcompeted and subsequently lost, resistance genes embedded on conjugative plasmids can persist or even increase in abundance, as a consequence of their sometimes extremely broad host ranges and high transfer frequencies [22, 45-48]. In controlled single strain experiments plasmid born resistance proved more costly than chromosomal resistance [7]. However, in more complex scenarios selection for mobile genetic element borne resistance usually depends not only on the single acquired resistance gene, but a combination of other linked traits encoded by the MGE as part of the communal gene pool [49]. Thus, difficulties in making general predictions on the selection dynamics of horizontally acquired resistance in microbial communities arise that merit future research efforts.

In summary, we show that selection for AMR was influenced by being embed in a 'natural' microbial community, such that the MSC was increased by more than one order of magnitude for two different antibiotics. Further to reducing relative fitness of resistance, being embedded in a community would also reduce absolute fitness, which has been argued to sometimes be the major driver of spread of resistance [50]. The aim of this study was to identify these general mechanisms underlying decreased selection for AMR in complex community context with a high degree of environmental and ecological realism. More specific individual interactions contributing to these general effects might however depend on the specific community, antibiotic and corresponding resistance genes and should be investigated in the future.

To determine MSCs that are relevant in environmental settings it is thus crucial to test for selection in a complex community context, rather than in single strain systems. Understanding under which concentrations selection for and thus long-term fixation of newly acquired resistance mechanisms is occurring is crucial for future mitigation of the spread of resistance genes as well as their potentially pathogenic hosts [51, 52]. Our results further stress the need to preferentially use narrow spectrum antibiotics in clinical therapy to maintain a healthy microbiome within the patient that can more easily recover after antibiotic administration [53], thus decreasing the likelihood of positive selection for pathogens that might have acquired resistance when embedded in a community.

Acknowledgements UK received funding from the European Union's Horizon 2020 research and innovation program under Marie Skłodowska-Curie grant agreement no. 751699. UK, AB and WG were supported through an MRC/BBSRC grant (MR/N007174/1). XY thanks The University of Hong Kong for a postgraduate studentship.

\section{Compliance with ethical standards}

Conflict of interest The authors declare that they have no conflict of interest.

Publisher's note: Springer Nature remains neutral with regard to jurisdictional claims in published maps and institutional affiliations.

Open Access This article is licensed under a Creative Commons Attribution 4.0 International License, which permits use, sharing, adaptation, distribution and reproduction in any medium or format, as long as you give appropriate credit to the original author(s) and the source, provide a link to the Creative Commons license, and indicate if changes were made. The images or other third party material in this article are included in the article's Creative Commons license, unless indicated otherwise in a credit line to the material. If material is not included in the article's Creative Commons license and your intended use is not permitted by statutory regulation or exceeds the permitted use, you will need to obtain permission directly from the copyright holder. To view a copy of this license, visit http://creativecommons. org/licenses/by/4.0/.

\section{References}

1. WHO. Antimicrobial Resistance Global Report on Surveillance. 2014. https://www.who.int/drugresistance/documents/surveilla ncereport/en/.

2. D'Costa VM, King CE, Kalan L, Morar M, Sung WWL, Schwarz C, et al. Antibiotic resistance is ancient. Nature. 2011;477:457-61.

3. Knapp CW, Dolfing J, Ehlert PAI, Graham DW. Evidence of increasing antibiotic resistance gene abundances in archived soils since 1940. Environ Sci Technol. 2010;44:580-7.

4. Drlica K. The mutant selection window and antimicrobial resistance. J Antimicrob Chemother. 2003;52:11-7.

5. Drlica K, Zhao X. Mutant selection window hypothesis updated. Clin Infect Dis. 2007;44:681-8.

6. Gullberg E, Cao S, Berg OG, Ilbäck C, Sandegren L, Hughes D, et al. Selection of resistant bacteria at very low antibiotic concentrations. PLoS Pathog. 2011;7:e1002158.

7. Gullberg E, Albrecht LM, Karlsson C, Sandegren L, Andersson DI. Selection of a multidrug resistance plasmid by sublethal levels of antibiotics and heavy metals. MBio. 2014;5:e01918-14.

8. Carlet J. The gut is the epicentre of antibiotic resistance. Antimicrob Resist Infect Control. 2012;1:39.

9. Lundström SV, Östman M, Bengtsson-Palme J, Rutgersson C, Thoudal M, Sircar T, et al. Minimal selective concentrations of tetracycline in complex aquatic bacterial biofilms. Sci Total Environ. 2016;553:587-95.

10. Murray AK, Zhang L, Yin X, Zhang T, Buckling A, Snape J, et al. Novel insights into selection for antibiotic resistance in complex microbial communities. MBio. 2018;9:e00969-18.

11. Liu A, Fong A, Becket E, Yuan J, Tamae C, Medrano L, et al. Selective advantage of resistant strains at trace levels of antibiotics: a simple and altrasensitive color test for detection of antibiotics and genotoxic agents. Antimicrob Agents Chemother. 2011;55:1204-10.

12. Sorg RA, Lin L, van Doorn GS, Sorg M, Olson J, Nizet V, et al. Collective resistance in microbial communities by intracellular antibiotic deactivation. PLoS Biol. 2016;14:e2000631.

13. Yurtsev EA, Chao HX, Datta MS, Artemova T, Gore J. Bacterial cheating drives the population dynamics of cooperative antibiotic resistance plasmids. Mol Syst Biol. 2013;9:683.

14. Medaney F, Dimitriu T, Ellis RJ, Raymond B. Live to cheat another day: bacterial dormancy facilitates the social exploitation of $\beta$-lactamases. ISME J. 2016;10:778-87. 
15. Cao J, Kürsten D, Schneider S, Knauer A, Günther PM, Köhler JM. Uncovering toxicological complexity by multi-dimensional screenings in microsegmented flow: modulation of antibiotic interference by nanoparticles. Lab Chip. 2012;12:474-84.

16. Churski K, Kaminski TS, Jakiela S, Kamysz W, Baranska-Rybak W, Weibel DB, et al. Rapid screening of antibiotic toxicity in an automated microdroplet system. Lab Chip. 2012;12:1629.

17. Kraaijeveld AR, Limentani EC, Godfray HCJ. Basis of the tradeoff between parasitoid resistance and larval competitive ability in Drosophila melanogaster. Proc Biol Sci. 2001;268:259-61.

18. Gómez P, Buckling A. Bacteria-phage antagonistic coevolution in soil. Science. 2011;332:106-9.

19. Tenaillon O, Skurnik D, Picard B, Denamur E. The population genetics of commensal Escherichia coli. Nat Rev Microbiol. 2010;8:207-17.

20. Remus-Emsermann MNP, Gisler P, Drissner D. MiniTn7transposon delivery vectors for inducible or constitutive fluorescent protein expression in Enterobacteriaceae. FEMS Microbiol Lett. 2016;363:fnw178.

21. Klümper U, Dechesne A, Smets BF. Protocol for evaluating the permissiveness of bacterial communities toward conjugal plasmids by quantification and isolation of transconjugants. In: McGenity T., Timmis K., Nogales B. editors. Hydrocarbon and lipid microbiology protocols, springer protocols handbook. Springer, Berlin, Heidelberg, 2014.

22. Klümper U, Riber L, Dechesne A, Sannazzarro A, Hansen LH, Sørensen SJ, et al. Broad host range plasmids can invade an unexpectedly diverse fraction of a soil bacterial community. ISME J. 2015;9:934-45.

23. Martínez-García E, Calles B, Arévalo-Rodríguez M, De Lorenzo V. PBAM1: an all-synthetic genetic tool for analysis and construction of complex bacterial phenotypes. BMC Microbiol. 2011;11:38.

24. Martínez-García E, Aparicio T, de Lorenzo V, Nikel PI. New transposon tools tailored for metabolic engineering of gram-negative microbial cell factories. Front Bioeng Biotechnol. 2014;2:46.

25. Kovach ME, Elzer PH, Steven Hill D, Robertson GT, Farris MA, Roop RM, et al. Four new derivatives of the broad-host-range cloning vector pBBR1MCS, carrying different antibioticresistance cassettes. Gene. 1995;166:175-6.

26. Großkopf T, Zenobi S, Alston M, Folkes L, Swarbreck D, Soyer OS. A stable genetic polymorphism underpinning microbial syntrophy. ISME J. 2016;10:2844-53.

27. Kozich JJ, Westcott SL, Baxter NT, Highlander SK, Schloss PD. Development of a dual-index sequencing strategy and curation pipeline for analyzing amplicon sequence data on the MiSeq Illumina sequencing platform. Appl Environ Microbiol. 2013;79:5112-20.

28. Schloss PD, Westcott SL, Ryabin T, Hall JR, Hartmann M, Hollister $\mathrm{EB}$, et al. Introducing mothur: open-source, platform-independent, community-supported software for describing and comparing microbial communities. Appl Environ Microbiol. 2009;75:7537-41.

29. Wang Q, Garrity GM, Tiedje JM, Cole JR. Naïve Bayesian classifier for rapid assignment of rRNA sequences into the new bacterial taxonomy. Appl Environ Microbiol. 2007;73:5261-7.

30. Bray JR, Curtis JT. An ordination of the upland forest communities of Southern Wisconsin. Ecol Monogr. 1957;27:325-49.

31. Gravina S, Vijg J. Epigenetic factors in aging and longevity. Pflugers Arch . 2010;459:247-58.

32. Anderson MJ. A new method for non-parametric multivariate analysis of variance. Austral Ecol. 2001;26:32-46.

33. Yang Y, Jiang X, Chai B, Ma L, Li B, Zhang A, et al. ARGsOAP: online analysis pipeline for antibiotic resistance genes detection from metagenomic data using an integrated structured ARG-database. Bioinformatics. 2016;32:2346-51.
34. Kirkpatrick S, Gelatt CD, Vecchi MP. Optimization by simulated annealing. Science. 1983;220:671-80.

35. Flach CF, Genheden M, Fick J, Joakim Larsson DG. A comprehensive screening of Escherichia coli isolates from Scandinavia's largest sewage treatment plant indicates no selection for antibiotic resistance. Environ Sci Technol. 2018;52:11419-28.

36. Andersson DI, Hughes D. Antibiotic resistance and its cost: is it possible to reverse resistance? Nat Rev Microbiol. 2010;8:260-71.

37. Andersson DI, Hughes D. Persistence of antibiotic resistance in bacterial populations. FEMS Microbiol Rev. 2011;35:901-11.

38. Wale N, Sim DG, Jones MJ, Salathe R, Day T, Read AF. Resource limitation prevents the emergence of drug resistance by intensifying within-host competition. Proc Natl Acad Sci. 2017;114:201715874

39. Song T, Park Y, Shamputa IC, Seo S, Lee SY, Jeon HS, et al. Fitness costs of rifampicin resistance in Mycobacterium tuberculosis are amplified under conditions of nutrient starvation and compensated by mutation in the $\beta^{\prime}$ subunit of RNA polymerase. Mol Microbiol. 2014;91:1106-19.

40. Kümmerer K. Antibiotics in the aquatic environment-a reviewpart II. Chemosphere. 2009;75:435-41.

41. Mah TF, Pitts B, Pellock B, Walker GC, Stewart PS, O'Toole GA. A genetic basis for Pseudomonas aeruginosa biofilm antibiotic resistance. Nature. 2003;426:306-10.

42. Drenkard E, Ausubel FM. Pseudomonas biofilm formation and antibiotic resistance are linked to phenotypic variation. Nature. 2002;416:740-3.

43. Webb CO, Ackerly DD, McPeek MA, Donoghue MJ. Phylogenies and community ecology. Annu Rev Ecol Syst. 2002;33:475-505.

44. Perron GG, Gonzalez A, Buckling A. Source-sink dynamics shape the evolution of antibiotic resistance and its pleiotropic fitness cost. Proc Biol Sci. 2007;274:2351-6.

45. Klümper U, Dechesne A, Riber L, Brandt KK, Gülay A, Sørensen SJ, et al. Metal stressors consistently modulate bacterial conjugal plasmid uptake potential in a phylogenetically conserved manner. ISME J. 2017;11:152-65.

46. Shintani M, Matsui K, Inoue JI, Hosoyama A, Ohji S, Yamazoe A, et al. Single-cell analyses revealed transfer ranges of incP-1, incP-7, and incP-9 plasmids in a soil bacterial community. Appl Environ Microbiol. 2014;80:138-45.

47. Musovic S, Klümper U, Dechesne A, Magid J, Smets BF. Longterm manure exposure increases soil bacterial community potential for plasmid uptake. Environ Microbiol Rep. 2014;6:125-30.

48. Arias-Andres M, Klümper U, Rojas-Jimenez K, Grossart HP. Microplastic pollution increases gene exchange in aquatic ecosystems. Environ Pollut. 2018;237:253-61.

49. Norman A, Hansen LH, Sørensen SJ. Conjugative plasmids: vessels of the communal gene pool. Philos Trans R Soc Lond B Biol Sci. 2009;364:2275-89.

50. Day T, Huijben S, Read AF. Is selection relevant in the evolutionary emergence of drug resistance? Trends Microbiol. 2015;23:126-33.

51. Larsson DGJ, Andremont A, Bengtsson-Palme J, Brandt KK, de Roda Husman AM, Fagerstedt P, et al. Critical knowledge gaps and research needs related to the environmental dimensions of antibiotic resistance. Environ Int. 2018;117:132-8.

52. Smalla K, Cook K, Djordjevic SP, Klümper U, Gillings M. Environmental dimensions of antibiotic resistance: assessment of basic science gaps. FEMS Microbiol Ecol. 2018;94:fiy191. https://doi.org/10.1093/femsec/fiy191.

53. Palleja A, Mikkelsen KH, Forslund SK, Kashani A, Allin KH, Nielsen T, et al. Recovery of gut microbiota of healthy adults following antibiotic exposure. Nat Microbiol. 2018;3:1255-65. 